\title{
Ferramentas de extração de características para análise estática de aplicativos Android
}

\author{
Jonas Pontes, Estevão Costa ${ }^{1}$, Vanderson Rocha ${ }^{1}$, Nicolas Neves ${ }^{1}$, \\ Eduardo Feitosa $^{1}$, Joner Assolin ${ }^{2}$, Diego Kreutz ${ }^{2}$ \\ ${ }^{1}$ Universidade Federal do Amazonas (UFAM) \\ ${ }^{2}$ Universidade Federal do Pampa (Unipampa) \\ \{pontes, ecc, nicolas.neves, efeitosa\}@icomp.ufam.edu.br, \\ vanderson@ufam.edu.br, \{jonerassolin.aluno, kreutz\}@unipampa.edu.br
}

\begin{abstract}
Resumo. Neste estudo, investigamos algumas das ferramentas utilizadas para a extração de características estáticas de aplicações Android, que são frequentemente utilizadas por métodos de detecção de malwares. Demonstramos que as ferramentas podem diferir quanto ao quantitativo e a apresentação dos dados extraídos, o que acaba condicionando sua aplicabilidade às necessidades específicas de cada projeto.
\end{abstract}

\section{Introdução}

O Android ocupa a maior fatia no mercado de sistemas operacionais para dispositivos móveis, estando presente em aproximadamente $73 \%$ do total de smartphones no mundo, em agosto de $2021^{1}$. Esse crescimento também o torna o alvo preferido dos criminosos cibernéticos, que produzem aplicações maliciosas (malwares) para atingir objetivos financeiros, sociais ou políticos [Pan et al., 2020, Bibi et al., 2020].

Para a detecção de malwares, as atividades iniciais são identificação e extração de características que possam levar a percepção se o aplicativo é ou não malicioso. Tipicamente, as técnicas utilizadas para extrair as características são as análises estática, dinâmica ou híbrida. Na análise estática, as características do Android Package (APK) são extraídas sem a necessidade de execução do aplicativo; na análise dinâmica, a extração de características é realizada a partir da execução da aplicação; e na híbrida, há a combinação dos dois tipos anteriores. A abordagem de análise estática é a mais utilizada para detecção de aplicativos maliciosos, pois consome menos tempo e recursos computacionais quando em comparação às outras técnicas [Wang et al., 2019].

A importância das características para o processo de detecção de malwares é facilmente compreendida quando se empregam soluções baseadas no aprendizado de máquina [Wang et al., 2019, Sharma and Rattan, 2021]. Isto porque, para o correto funcionamento, essas soluções (e seus modelos) dependem de conjuntos de dados (datasets) corretos e atualizados, requerendo assim a extração detalhada de características dos aplicativos [Dharmalingam and Palanisamy, 2021, Alazab et al., 2020, Zhang et al., 2020].

Neste trabalho objetivamos avaliar empiricamente ferramentas de extração de características estáticas de APKs, buscando identificar aquelas que melhor se ajustam à extração de caraterísticas para a construção de datasets atualizados para detecção de

1 https://gs.statcounter.com/os-market-share/mobile/worldwide 
malwares Android. Para a avaliação selecionamos 7 ferramentas e submetemos 6 delas a um processo de extração de características de aplicativos rotulados como benignos e maliciosos, de diferentes tamanhos e diferentes níveis de APIs Android. Como contribuições, podemos destacar: (a) identificação das ferramentas com o melhor desempenho para extração de características de APKs; e (b) análise e discussão de questões de usabilidade e exigências computacionais das ferramentas avaliadas.

O restante do trabalho está organizado da seguinte forma. Na Seção 2 apresentamos a metodologia para a seleção e avaliação de ferramentas. Nas Seções 3 e 4 discutimos os resultados e apresentamos as considerações finais, respectivamente.

\section{Metodologia}

O processo de seleção e avaliação das ferramentas foi composto pelas seguintes etapas: (i) busca por ferramentas de extração; (ii) escolha das ferramentas de interesse; (iii) definição dos aplicativos a serem testados; e (iv) avaliação qualitativa das ferramentas. Na primeira etapa, realizamos uma busca por ferramentas de extração de características estáticas de aplicativos Android, em que as revisões sistemáticas (e.g., [Faruki et al., 2014, Hijawi et al., 2021, Schmicker et al., 2018]) e documentos técnicos específicos da áre $2^{2}$ foram o ponto de partida.

A partir da lista inicial de 33 ferramentas, na segunda etapa, avaliamos a admissibilidade de cada ferramenta. Como critério de inclusão, definimos a capacidade de extração a partir do APK e a geração de uma saída estruturada, da qual fosse possível identificarmos as características coletadas. Admitimos, igualmente, ferramentas de engenharia reversa, i.e., que tem como saída os arquivos originais que compõem o APK, incluindo o de manifesto (AndroidManifest.xml), a partir do qual podemos facilmente extrair características. Como principais critérios de exclusão, definimos: (i) a capacidade de extrair características dos APKs e (ii) estar disponível para qualquer pessoa utilizar sem restrições ou custos financeiros. Assim, ferramentas que fazem tipicamente parte do processo de detecção de malwares (e.g., conversores de arquivos .class para Java, visualizadores de arquivos binários, ferramentas de teste de penetração), mas não apresentam uma saída com as características do APK, foram eliminadas.

A partir da leitura das documentações ou artigos, realizada por no mínimo dois coautores, excluímos 26 ferramentas da lista inicial. As 7 ferramentas selecionadas (Androguard 4 , PSCout $1^{5}$, Android Decompiler ${ }^{6}$, AndroParse ${ }^{7}$, PHP APK Parser ${ }^{8}$, Mobile Audit ${ }^{9}$ e Apktoo 10 estão resumidas na Tabela 1

As dependências para instalação (e.g., comandos, bibliotecas, linguagens) de cada uma das ferramentas estão listadas na Tabela 2 .

\footnotetext{
2 https://github.com/ashishb/android-security-awesome

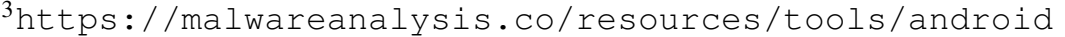

4 https://github.com/androguard/androguard

5 https://github.com/dlgroupuoft/Pscout

6 https://github.com/dirkvranckaert/AndroidDecompiler

/https://github.com/rschmicker/Androparse

8 https://github.com/iwinmin/php_apk_parser

sthtps://github.com/mpast/mobileAudit

10 https://ibotpeaches.github.io/Apktool
} 
Tabela 1. Ferramentas para descompactação de arquivos selecionadas

\begin{tabular}{l|l|l}
\hline \hline Ferramenta & Entradas & Saídas \\
\hline \multirow{2}{*}{$\begin{array}{l}\text { Androguard } \\
\text { PScout }\end{array} \begin{array}{l}\text { Arquivos .dex, .odex, .apk } \\
\text { Android Decompiler }\end{array}$} & $\begin{array}{l}\text { Permissões, componentes do APP, AndroidManifest.xml, arquivos .dex, } \\
\text { gráfico de fluxo de controle, intents, código de operação, classes }\end{array}$ \\
\cline { 2 - 3 } & AndroidManifest.xml & Permissões \\
\cline { 2 - 3 } $\begin{array}{l}\text { AndroParse } \\
\text { PHP APK Parser }\end{array}$ & $\begin{array}{l}\text { Arquivos do APK, incluindo AndroidManifest.xml, código-fonte, arqui- } \\
\text { vos de recursos }\end{array}$ \\
\cline { 2 - 3 } $\begin{array}{l}\text { Mobile Audit } \\
\text { Apktool }\end{array}$ & Arquivo .apk & APIs, intents e permissões \\
\cline { 2 - 3 } & Arquivo .apk & $\begin{array}{l}\text { AndroidManifest.xml, permissões, componentes do APP, intents, clas- } \\
\text { ses. }\end{array}$ \\
\cline { 2 - 3 } & Arquivo .apk & $\begin{array}{l}\text { Permissões, componentes do APP e outras strings } \\
\text { Arquivos do APK, incluindo AndroidManifest.xml, recursos e clas- }\end{array}$ \\
\hline
\end{tabular}

Tabela 2. Dependências das ferramentas

\begin{tabular}{l|l}
\hline \hline Ferramenta & Dependências \\
\hline Androguard & Python 3, pip, dependências do pacote “androguard": asn1crypto, click, colorama, future, ipython, \\
& lxml, matplotlib, networkx, pydot e pygments \\
Android Decompiler & Java \\
AndroParse & Go, Glide, Android Asset Packaging Tool (AAPT), Java e make \\
Apktool & Java \\
Mobile Audit & Docker e Docker compose \\
PHP APK Parser & PHP, composer, php7.2-zip, php-mbstring e php-simplexml \\
PScout & Perl, Java e XML::Simple \\
\hline
\end{tabular}

É importante relatar que a ferramenta AndroParse não foi avaliada como as demais em razão da falta de informações necessárias para instalação e execução. Não existe especificação das versões de suas dependências, bem como os comandos de configuração da ferramenta (make) e de teste da instalação (make unittest) apresentam erros em diversos ambientes. Testes de instalação da AndroParse, sem sucesso, foram realizados nos sistemas operacionais Windows 10 e Linux (Ubuntu 20.04 LTS, Kernel 5.11.0-27generic, 64 bits e também nas versões Ubuntu 18.04 LTS e 16.04 LTS), por 5 co-autores (avaliadores). A descrição completa dos ambientes de teste do AndroParse e das demais ferramentas estão disponíveis no endereço de documentação dos sistemas operacionais ${ }^{11}$.

Para avaliar o processo de extração e sua qualidade, definimos um conjunto de aplicativos benignos e malignos (terceira etapa), em igual proporção, e com diferentes níveis de APIs Android, principalmente as mais recentes. Neste trabalho, utilizamos 10 aplicativos, sendo cinco benignos (B) e igual quantidade de malwares $(\mathrm{M})$, contemplando os níveis de API Android 28, 29 e 30. Utilizamos o Google Play Store e o repositório ${ }^{12}$ desenvolvido por [Wang et al., 2021] como fonte dos APKs benignos e maliciosos, nesta ordem. A seleção ocorreu de forma aleatória, dentro do critério de APIs de níveis 2830, isto é, APIs mais recentes do Android. A Tabela 3 lista os aplicativos selecionados, incluindo informações sobre o tamanho, versões da API e resumo criptográfico.

Por fim, para avaliar qualitativamente as ferramentas (quarta etapa), consideramos os seguintes procedimentos: (i) definir as características de interesse; (ii) submeter diferentes aplicativos, maliciosos e benignos a cada ferramenta; (iii) e comparar as saídas produzidas. A avaliação de uma ferramenta de extração de características de aplicativos

11 https://github.com/Malware-Hunter/ExternalTools/blob/main/wrseg21

12 https://zenodo.org/record/4660140\#.YUEZURmSmUl 
Tabela 3. Aplicativos considerados nesta pesquisa

\begin{tabular}{|c|c|c|c|}
\hline Nome & Resumo Criptográfico (SHA256) & $\mathbf{N}^{0}$ Bytes & API \\
\hline Cartola FC $(B)$ & aeda946f4099638ab800d19c13d94b66d1615d3a72d3388415476ae9eb2519e2 & 34.424 .777 & 29 \\
\hline $\operatorname{Instagram}(B)$ & c3bf7adf8403cee6ba7bdfa5a3fbf105a9071c21fa19d3c8d480cdf6f609d2a2 & 40.166 .591 & 30 \\
\hline $\operatorname{IPTV}(B)$ & 28da45ae147f377719f3d3178aa14c22df4fc761b13193367c6f5ba82fe7899d & 22.460 .779 & 28 \\
\hline Spotify $(B)$ & e4986faf1c91f10574c252212bec22c8a51446ba30e2ec3b1705c0d25b635cd9 & 30.940 .271 & 29 \\
\hline WhatsApp $(B)$ & 454d0a18ff06084a952a709247360558717b7e6537d3b2637b0007691cee456f & 33.727 .503 & 29 \\
\hline Tracking COVID-19 $(M)$ & 0aa1f7e03588373e3520256d43df0f3ce32f5c0eed5c90910a2f5babb8911fff & 3.172 .731 & 29 \\
\hline Covid19 Symptom Tracker $(M)$ & 0b2131a3d58e6980496ea8b26ee18c72fa08389750d7812180ce459946099841 & 1.849 .005 & 28 \\
\hline COVID-RD $(M)$ & 0ba773c0bffa04c9f6a6216667873cce339bda28646d267f6515b6bce4cc36d0 & 52.267 .255 & 28 \\
\hline COVID-19 $(M)$ & 0d2b8232bf5e40deea3acf91c398e4a34085a06f3ef9fa109eefdc954661c5df & 7.821 .886 & 28 \\
\hline Corona Help $(M)$ & $1 \mathrm{~b} 3207137156 \mathrm{f} 94076 \mathrm{~d} 85 \mathrm{c} 67638 \mathrm{e} 561 \mathrm{~b} 366 \mathrm{a}$ bbf5225c50e525c3ed5df0cd317 & 25.148 .214 & 29 \\
\hline
\end{tabular}

Android pode considerar quais características ela é capaz de obter, bem como a quantidade coletada. Neste trabalhos avaliamos as ferramentas considerando as características permissões, intents, API, componentes do APP e opcode, as quais são as mais utilizadas, como características estáticas, pelos trabalhos levantados por [Wang et al., 2019].

\section{Resultados e discussões}

\subsection{Análise das ferramentas de extração}

Para mensurar a capacidade em extrair características estáticas dos APK, consideramos, inicialmente, a soma de cada característica extraída nos cinco APKs maliciosos ( $M$.) e nos cinco benignos $(B$.$) . Nesta análise, apenas as 4$ ferramentas de extração de características foram consideradas. A Tabela 4 apresenta o resultado da extração de características, considerando as características permissões, API, intents, componentes do APP e opcode ${ }^{13}$.

Tabela 4. Resultado da extração de características estáticas

\begin{tabular}{l|l|l|l|l|l|l|l|l|l|l}
\hline \hline & \multicolumn{3}{|l}{ Permissões } & \multicolumn{1}{c}{ Intents } & \multicolumn{1}{c}{ API } & \multicolumn{2}{c}{ Componentes do APP } \\
\cline { 2 - 10 } Ferramenta & B. & M. & B. & M. & B. & M. & B. & M. & B. & M. \\
\hline Androguard & 144 & 43 & 292 & 53 & 3470999 & 658404 & 934 & 91 & 13376816 & 2614284 \\
PHP APK Parser & 140 & 39 & 74 & 13 & 0 & 0 & $565^{* *}$ & $46^{* *}$ & 0 & 0 \\
Mobile Audit & 144 & 33 & $*$ & $*$ & 0 & 0 & $846^{*}$ & $91^{*}$ & 0 & 0 \\
PScout & 9 & 0 & 0 & 0 & 0 & 0 & 0 & 0 & 0 & 0 \\
\hline
\end{tabular}

Como podemos observar, Androguard é a ferramenta que obteve o melhor desempenho em relação a extração das características. É importante destacarmos que a documentação da ferramenta informa que há suporte apenas até a API 29 do Android (APIs superiores são tratadas como 29). Para todas as características analisadas, a ferramenta apresenta valores iguais ou superiores às demais.

Mobile Audit foi a ferramenta que obteve resultados mais próximos do Androguard quanto à extração de permissões. Entretanto, não apresentou resultados para API e opcode. Ademais, vale destacar que o conjunto de características que formam os componentes do aplicativo no Mobile Audit difere daquele apresentado por [Wang et al., 2019]

\footnotetext{
${ }^{13} \mathrm{Um}$ OpCode (Código de Operação) é uma instrução única (atômica) que pode ser executada pela CPU. No caso do Android, opcodes são as instruções da máquina virtual Dalvik para execução do aplicativo.
} 
- que é o mesmo apresentado na análise componentes de aplicativo extraídos via Androguard, ou seja, além de activity, service, broadcast receiver e content provider, a ferramenta considera intents como parte de componentes do aplicativo, não sendo possível isolar as intents dentro do conjunto apresentado (destacado por $\star$ na Tabela 4).

A ferramenta PHP APK Parser foi capaz de extrair permissões em quantidade próxima àquelas obtidas via Androguard e Mobile Audit. Por outro lado, extraiu bem menos intents que a Androguard. Quanto aos componentes do aplicativo, somente é capaz de extrair activities (denotado por $\star \star$ na Tabela 4). As demais características de interesse deste trabalho não são extraíveis através desta ferramenta.

A PScout, apesar de obter características de aplicações Android, não o faz a partir de um APK, como as demais ferramentas citadas. Ele necessita do arquivo de manifesto do aplicativo e neste trabalho utilizamos o Apktool para obtê-lo do APK. Os resultados conseguidos a partir da ferramenta são modestos se comparados aos das demais ferramentas. Destacadamente, não há obtenção de nenhuma característica de aplicação maliciosa. Analisando o código-fonte da ferramenta, notamos que a ferramenta retorna apenas permissões cuja tag XML "permission" possui o atributo "protectionLevel" com o valor de "signature" ou "signatureOrSystem", ou que esteja combinado com a expressão regular Perl "m/system/i". Desse modo, o parser não retorna todas as permissões existentes em um arquivo AndroidManifest.xml, pois permissões podem ser declaradas em outras tags XML (e.g., "uses-permission" e "uses-permission-sdk-23").

\subsection{Análise das ferramentas de engenharia reversa}

Com relação as ferramentas de engenharia reversa, Android Decompiler executou com falhas e não realizou a extração dos arquivos originais do APKs. A causa dessa falha é o fato dessa ferramenta se tratar de um shell script que utiliza outras ferramentas de engenharia reversa em versões antigas, que não são mais mantidas ou atualizadas nos sistemas operacionais atuais. O repositório da ferramenta no GitHub não é atualizado desde Outubro de 2014. Além disso, consta em seu repositório que a formatação de código do shell script não tem suporte para todas as plataformas, e que foi construído para MacOS, mas que a maior parte dele deve funcionar nos ambientes UNIX. Portanto, devido a este suporte limitado e aos erros obtidos durante os testes, notamos que a ferramenta Android Decompiler não é mais (atualmente) uma alternativa viável para realizar engenharia reversa de APKs.

A ferramenta Apktool, por outro lado, mostrou-se mais completa para engenharia reversa, e que ao ser combinada com um parser, possibilita a extração de diversas características. Portanto, supera o Android Decompiler em questão de utilização e viabilidade técnica. Podemos destacar também que a ferramente tem um repositório vasto de versões, sendo a versão mais atual do ano de 2021. Os resultados sustentam o motivo pelo qual ela é frequentemente utilizada em trabalhos que necessitam de engenharia reversa.

\section{Considerações Finais}

Neste trabalho, apresentamos uma avaliação qualitativa de ferramentas de extração de características estáticas e engenharia reversa de aplicativos Android, considerando permissões, API, intents, componentes de aplicativo e opcode como características-alvo. Os resultados mostram que, dentre as ferramentas consideradas, Androguard apresenta os 
melhores resultados quanto à extração de características, capturando dados para todas as características consideradas, mesmo que para isso demande um tempo maior para a análise do APK. Mobile Audit e e PHP APK Parser também apresentaram bons resultados para extração de permissões, mas os resultados deixam a desajar se considerarmos todas as características. Quanto à ferramentas de engenharia reversa, Apktool entregou aquilo que se espera: é uma ferramenta atualizada e que exporta os arquivos do APK. Portanto, resumidamente, nosso estudo indica que Apktool e Androguard são ferramentas recomendáveis para engenharia reversa e extração de características, respectivamente.

Como trabalhos futuros, podemos citar: (a) ampliar a pesquisa, considerando um conjunto maior de ferramentas e APKs; (b) comparar as ferramentas quantitativamente, considerando uso do processador, memória e tempo de execução; (c) construir um dataset para detecção de malwares Android utilizando as ferramentas destacadas.

\section{Agradecimento}

O presente trabalho foi realizado com apoio da Coordenação de Aperfeiçoamento de Pessoal de Nível Superior - Brasil (CAPES) - Código de Financiamento 001 e financiado, conforme previsto nos Arts. 21 e 22 do decreto $n^{\circ} 10.521 / 2020$, nos termos da Lei Federal $\mathrm{n}^{\circ}$ 8.387/1991, através de convênio nº03/2021, firmado entre ICOMP/UFAM, Flextronics da Amazônia Ltda e Motorola Mobility Comércio de Produtos Eletrônicos Ltda.

\section{Referências}

Alazab, M., Alazab, M., Shalaginov, A., Mesleh, A., and Awajan, A. (2020). Intelligent mobile malware detection using permission requests and api calls. Future Generation Computer Systems, 107:509-521.

Bibi, I., Akhunzada, A., Malik, J., Iqbal, J., Musaddiq, A., and Kim, S. (2020). A dynamic dl-driven architecture to combat sophisticated android malware. IEEE Access, 8:129600-129612.

Dharmalingam, V. P. and Palanisamy, V. (2021). A novel permission ranking system for android malware detection-the permission grader. Journal of Ambient Intelligence and Humanized Computing, 12(5):5071-5081.

Faruki, P., Bharmal, A., Laxmi, V., Ganmoor, V., Gaur, M. S., Conti, M., and Rajarajan, M. (2014). Android security: a survey of issues, malware penetration, and defenses. IEEE communications surveys \& tutorials, 17(2):998-1022.

Hijawi, W., Alqatawna, J., Al-Zoubi, A. M., Hassonah, M. A., and Faris, H. (2021). Android botnet detection using machine learning models based on a comprehensive static analysis approach. Journal of Information Security and Applications, 58:102735.

Pan, Y., Ge, X., Fang, C., and Fan, Y. (2020). A systematic literature review of android malware detection using static analysis. IEEE Access, 8:116363-116379.

Schmicker, R., Breitinger, F., and Baggili, I. (2018). Androparse-an android feature extraction framework and dataset. In International Conference on Digital Forensics and Cyber Crime, pages 66-88. Springer.

Sharma, T. and Rattan, D. (2021). Malicious application detection in android - a systematic literature review. Computer Science Review, 40:100373.

Wang, L., He, R., Wang, H., Xia, P., Li, Y., Wu, L., Zhou, Y., Luo, X., Sui, Y., Guo, Y., and Xu, G. (2021). Beyond the virus: A first look at coronavirus-themed mobile malware.

Wang, W., Zhao, M., Gao, Z., Xu, G., Xian, H., Li, Y., and Zhang, X. (2019). Constructing features for detecting android malicious applications: Issues, taxonomy and directions. IEEE Access, 7:6760267631.

Zhang, X., Zhang, Y., Zhong, M., Ding, D., Cao, Y., Zhang, Y., Zhang, M., and Yang, M. (2020). Enhancing state-of-the-art classifiers with api semantics to detect evolved android malware. In Proceedings of the 2020 ACM SIGSAC, CCS '20, page 757-770, New York, NY, USA. ACM. 